\title{
The venus kinase receptor (VKR) family: structure and evolution
}

\author{
Mathieu Vanderstraete ${ }^{1}$, Nadège Gouignard ${ }^{1}$, Arnaud Ahier $^{2}$, Marion Morel', Jérôme Vicogne ${ }^{3}$ \\ and Colette Dissous ${ }^{1 *}$
}

\begin{abstract}
Background: Receptor tyrosine kinases (RTK) form a family of transmembrane proteins widely conserved in Metazoa, with key functions in cell-to-cell communication and control of multiple cellular processes. A new family of RTK named Venus Kinase Receptor (VKR) has been described in invertebrates. The VKR receptor possesses a Venus Fly Trap (VFT) extracellular module, a bilobate structure that binds small ligands to induce receptor kinase activity. VKR was shown to be highly expressed in the larval stages and gonads of several invertebrates, suggesting that it could have functions in development and/or reproduction.
\end{abstract}

Results: Analysis of recent genomic data has allowed us to extend the presence of VKR to five bilaterian phyla (Platyhelminthes, Arthropoda, Annelida, Mollusca, Echinodermata) as well as to the Cnidaria phylum. The presence of NveVKR in the early-branching metazoan Nematostella vectensis suggested that VKR arose before the bilaterian radiation. Phylogenetic and gene structure analyses showed that the 40 receptors identified in 36 animal species grouped monophyletically, and likely evolved from a common ancestor. Multiple alignments of tyrosine kinase (TK) and VFT domains indicated their important level of conservation in all VKRs identified up to date. We showed that VKRs had inducible activity upon binding of extracellular amino-acids and molecular modeling of the VFT domain confirmed the structure of the conserved amino-acid binding site.

Conclusions: This study highlights the presence of VKR in a large number of invertebrates, including primitive metazoans like cnidarians, but also its absence from nematodes and chordates. This little-known RTK family deserves to be further explored in order to determine its evolutionary origin, its possible interest for the emergence and specialization of Metazoa, and to understand its function in invertebrate development and/or reproductive biology.

\section{Background}

Receptor Tyrosine Kinases (RTKs) are transmembrane proteins that are involved in many fundamental intra- and inter-cellular processes. RTKs have essential multicellular-specific functions, including cell-to-cell communications, control of cell proliferation and differentiation [1]. They have been found in all metazoan genomes, from the marine sponge Geodia cydonium to humans [2,3]. Moreover, RTKs were also shown to be present in choanoflagellates [4,5] and Filasterea [6], which are the sister groups of Metazoa. A large number of RTKs are conserved throughout evolution, but unique

\footnotetext{
* Correspondence: colette.dissous@pasteur-lille.fr

${ }^{1}$ Center for Infection and Immunity of Lille (CIIL), Inserm U1019, CNRS-UMR

8204, Institut Pasteur de Lille, 59019, Lille, France

Full list of author information is available at the end of the article
}

and organism-specific RTKs have been identified, such as Sweet tooth in Hydra vulgaris [7] or kin15/kin16 in Caenorhabditis elegans [8,9]. RTKs have been classified into distinct families, depending on the modular composition of their extracellular domains and their ability to bind different types of ligands, as well as by their kinase domain sequences. The human genome encodes 58 RTKs, and these receptors are classified into 20 families [10]. According to the data regrouped in http://kinase.com, the invertebrate model organisms Drosophila melanogaster and C. elegans possess 16 and 29 RTKs and share 11 and 10 families with human RTKs, respectively [3].

Venus Kinase receptors (VKRs) constitute an RTK family, originally found in the parasite platyhelminth Schistosoma mansoni [11], then in several other invertebrates (insects and echinoderms). However, no VKR

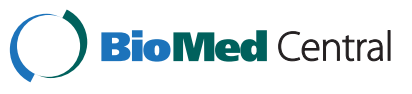


could be found in any chordate genome and, more strikingly, this receptor was not present in C. elegans and $D$. melanogaster [12]. VKR proteins possess an atypical structure [11,12], containing an intracellular tyrosine kinase (TK) domain similar to that of insulin receptors, and an extracellular Venus Flytrap (VFT) domain. VFTs were first identified as bacterial periplasmic-binding proteins involved in the transport of small molecules, such as amino acids, sugars or ions and they constitute the binding pocket of different receptor types such as class C G-protein coupled receptors [13]. The VFT domain of VKR is related to that of the ANF receptor in protein family databases (pfam 01094) [12]. VKR kinases can be activated following the binding of amino-acids to the extracellular VFT domain [12,14], and this opens interesting perspectives on a novel mechanism for RTK activation as well as on possible specific and new functions of these receptors in cellular signalling.

In this paper, we present an up-dated version of the VKR family, resulting from an exhaustive research of VKR orthologs in the published genomes of a variety of organisms belonging to major phyla in Metazoa. We show that $v k r$ genes are present at least in five major phyla in Bilateria (Platyhelminthes, Arthropoda, Annelida, Mollusca, Echinodermata), and also, more strikingly, in the cnidarian Nematostella vectensis. Phylogenetic analyses indicated that all the putative protein sequences grouped monophyletically, and a new version of the VKR phylogeny was built. In silico analyses and multiple alignments of the VFT and TK functional domains of VKRs allowed us to reinforce the structural model of the receptor and to get a better prediction of potential ligands and kinase activity of VKRs.

\section{Results and discussion}

A large distribution of $v k r$ genes in eumetazoan genomes Previous studies have shown that VKRs constitute a distinct RTK family. These receptors were described in 15 different protostomes, including insects and the platyhelminth S. mansoni. A $v k r$ gene was also found in the deuterostome Strongylocentrotus purpuratus [12]. The aim of this study was to extend the VKR family to a large number of phyla in order to evaluate the place of these receptors in the animal kingdom and to contribute to a better understanding of their evolution.

Using BLAST approaches, we searched for $v k r$ genes in multiple genomic databases (see Methods). Results showed that $v k r$ genes were present in at least 36 species from six distinct phyla (Cnidaria, Echinodermata, Platyhelminthes, Mollusca, Annelida and Arthropoda, see Table 1). The protein architecture of each putatively encoded VKR was analysed using the SMART online software http://smart.embl-heidelberg.de/ in order to verify that newly discovered genes effectively encoded struc- turally conserved proteins, composed of an extracellular VFT module and an intracellular TK domain linked together by a single transmembrane $\alpha$-helix. In Platyhelminthes, two putative $v k r$ genes were detected in both Clonorchis sinensis and S. mansoni [14] parasitic trematodes. By contrast, only one $v k r$ was found in the genome of the three parasitic cestodes, Echinococcus multilocularis, Echinococcus granulosus and Hymenolepis microsto$m a$. No unequivocal VKR sequence could be found in the genome of the planarian Schmidtea mediterranea, but a sequence encoding a polypeptide composed of a TK domain and a truncated VFT module (GenBank: AAWT01078636.1) was identified, suggesting that a $v k r$ gene might still exist in Turbellaria. However, deeper investigations are needed to extend reliably the existence of VKR to planarian species and to the whole of the Platyhelminth phylum. Several new $v k r$ genes were also found in diverse insect genomes. We identified 15 new $v k r$ genes in 13 species belonging to the Hymenoptera and Lepidoptera orders. In Hymenoptera, on top of the two genes previously characterized in the honeybee Apis mellifera and in the parasitic wasp Nasonia vitripennis [12], we were able to identify 11 new $v k r$ genes in the genomes of eight Formicidae and three Apidae species. In Lepidoptera, we could show that both the silkworm Bombyx mori and the monarch butterfly Danaus plexippus possess two $v k r$ genes located in tandem on a single scaffold (GenBank accession numbers DF090406.1 and JH386161.1, respectively). Additionally, we could find truncated $v k r$ sequences in other arthropod genomes, such as those of Rhodnius prolixus (Hemiptera), Glossina morsitans morsitans (Diptera) and Daphnia pulex (Cladocera). However, the bad scores of similarity registered from sequence alignments and the lack of protein structure conservation led us to exclude these sequences from further phylogenetic analyses. These were performed to assess that the predicted proteins were VKR orthologs belonging to the same family. A maximum likelihood phylogenetic tree was generated under the JTT+I+G model with the support of three outgroups composed respectively of insulin (outgroup 1), RTK-like orphan (ROR) (outgroup 2) and epidermal growth factor (EGF) (outgroup 3) receptors of invertebrates (Figure 1). Results showed that all VKR sequences consistently form a monophyletic group distinct from the three other RTK families. Inside of the VKR family, distinct and robust groups were formed by hymenopteran, coleopteran or dipteran sequences. Platyhelminth VKRs form a cluster made of two distinct cestode and trematode branches. In the trematode branch, VKR1 proteins grouped distinctly from the VKR2 ones. Similarly, lepidopteran VKR1 and VKR2 sequences (BmVKR1/2 and DpVKR1/2) were split into two different branches, but surprisingly lepidopteran VKR1 proteins were rejected out of the arthropod group and placed at the root of the VKR 
Table 1 Complete list of the $\mathbf{4 0} \mathbf{v k r}$ genes found in genomic databases

\begin{tabular}{|c|c|c|c|c|}
\hline Species & Class & Name & Accession number & Database \\
\hline Acromyrmex echiniator & Insect & AeVKR & GL888498.1 & http://flybase.org/ \\
\hline Aedes aegypti & Insect & AaVKR & DAA06509.1 & http://blast.ncbi.nlm.nih.gov/Blast.cgi \\
\hline Anopheles gambiae & Insect & AgVKR & ACF34410.1 & http://blast.ncbi.nlm.nih.gov/Blast.cgi \\
\hline Apis florea & Insect & AfVKR & GL576580.1 & http://flybase.org/ \\
\hline Apis mellifera & Insect & AmVKR & ACF34409.1 & http://blast.ncbi.nlm.nih.gov/Blast.cgi \\
\hline Atta cephalotes & Insect & AcVKR & GL377380.1 & http://flybase.org/ \\
\hline Bombus impatiens & Insect & BiVKR & XP_003486761.1 & http://ncbi.nlm.nih.gov/ \\
\hline Bombus terrestris & Insect & BtVKR & GL898830.1 & http://flybase.org/ \\
\hline Bombyx mori & Insect & BmVKR1 & DF090406.1 & http://flybase.org/ \\
\hline Bombyx mori & Insect & BmVKR2 & DF090406.1 & http://flybase.org/ \\
\hline Camponotus floridanus & Insect & CfVKR & EFN73169.1 & http://ncbi.nlm.nih.gov/ \\
\hline Capitella teleta & Annelid & CtVKR & 136189 & $\begin{array}{l}\text { http://genome.jgi.doe.gov/pages/search-for-genes.jsf? } \\
\text { organism=Capcal }\end{array}$ \\
\hline Clonorchis sinensis & Trematode & CsVKR1 & GAA27163.2 & http://ncbi.nlm.nih.gov/ \\
\hline Clonorchis sinensis & Trematode & CSVKR2 & GAA49307.1 & http://ncbi.nlm.nih.gov/ \\
\hline Culex quinquefasciatus & Insect & CqVKR & DAA06510.1 & http://ncbi.nlm.nih.gov/ \\
\hline Danaus plexippus & Insect & DplVKR1 & EHJ69301.1 & http://ncbi.nlm.nih.gov/ \\
\hline Drosophila ananassae & Insect & DaVKR & DAA06508.1 & http://ncbi.nlm.nih.gov/ \\
\hline Drosophila grimshawi & Insect & DgVKR & DAA06505.1 & http://ncbi.nlm.nih.gov/ \\
\hline Drosophila mojavensis & Insect & DmoVKR & DAA06504.1 & http://ncbi.nlm.nih.gov/ \\
\hline Drosophila persimilis & Insect & DpVKR & DAA06507.1 & http://ncbi.nlm.nih.gov/ \\
\hline Drosophila pseudoopscura & Insect & DpseVKR & ACF34407.1 & http://ncbi.nlm.nih.gov/ \\
\hline Drosophila virilis & Insect & DVVKR & DAA06503.1 & http://ncbi.nlm.nih.gov/ \\
\hline Drosophila wilistoni & Insect & DWVKR & DAA06506.1 & http://ncbi.nlm.nih.gov/ \\
\hline Echinococcus granulosus & Cestode & EgVKR & NODE_166072 & $\begin{array}{l}\text { http://www.sanger.ac.uk/resour...s/echinococcus-granulosus. } \\
\text { html }\end{array}$ \\
\hline $\begin{array}{l}\text { Echinococcus } \\
\text { multilocularis }\end{array}$ & Cestode & EmVKR & pathogen_EMU_scaffold_007728 & $\begin{array}{l}\text { http://www.sanger.ac.uk/resour...s/echinococcus-multilocularis } \\
\text { html }\end{array}$ \\
\hline Harpegnathos saltator & Insect & HsVKR & EFN85558.1 & http://ncbi.nlm.nih.gov/ \\
\hline Hymenolepis microstoma & Cestode & HmVKR & 744 & $\begin{array}{l}\text { http://www.sanger.ac.uk/resour...hs/hymenolepis-microstoma. } \\
\text { html }\end{array}$ \\
\hline Linepithema humile & Insect & LhVKR & GL905323.1 & http://flybase.org/ \\
\hline Lottia gigantea & Mollusc & LgVKR & 109151 & $\begin{array}{l}\text { http://genome.jgi-psf.org/pages/search-for-genes.jsf? } \\
\text { organism=Lotgi1 }\end{array}$ \\
\hline Megachile rotundata & Insect & MrVKR & GL985818.1 & http://flybase.org/ \\
\hline Nasonia vitripennis & Insect & NVVKR & DAA06502.1 & http://ncbi.nlm.nih.gov/ \\
\hline Nematostella vectensis & Anthozoan & NveVKR & SB_43850 & http://nematostella.bu.edu/stellabase/ \\
\hline $\begin{array}{l}\text { Pediculus humanus } \\
\text { corporis }\end{array}$ & Insect & PhcVKR & DAA06501.1 & http://ncbi.nlm.nih.gov/ \\
\hline Pogonomyrmex barbatus & Insect & PbVKR & GL738256.1 & http://flybase.org/ \\
\hline Schistosoma mansoni & Trematode & SmVKR1 & AAL67949.1 & http://ncbi.nlm.nih.gov/ \\
\hline Schistosoma mansoni & Trematode & SmVKR2 & ADD91576.1 & http://ncbi.nlm.nih.gov/ \\
\hline Solenopsis invicta & Insect & SiVKR & $E F Z 12829.1$ & http://ncbi.nlm.nih.gov/ \\
\hline $\begin{array}{l}\text { Strongylocentrotus } \\
\text { purpuratus }\end{array}$ & Echinoidea & SpVKR & DAA06500 & http://ncbi.nlm.nih.gov/ \\
\hline Tribolium castaneum & Insect & TcVKR & ACF34408.1 & http://ncbi.nlm.nih.gov/ \\
\hline
\end{tabular}

Vkr gene name is specified for each species, as well as protein or scaffold accession number and website link of the database in which the gene was found. 


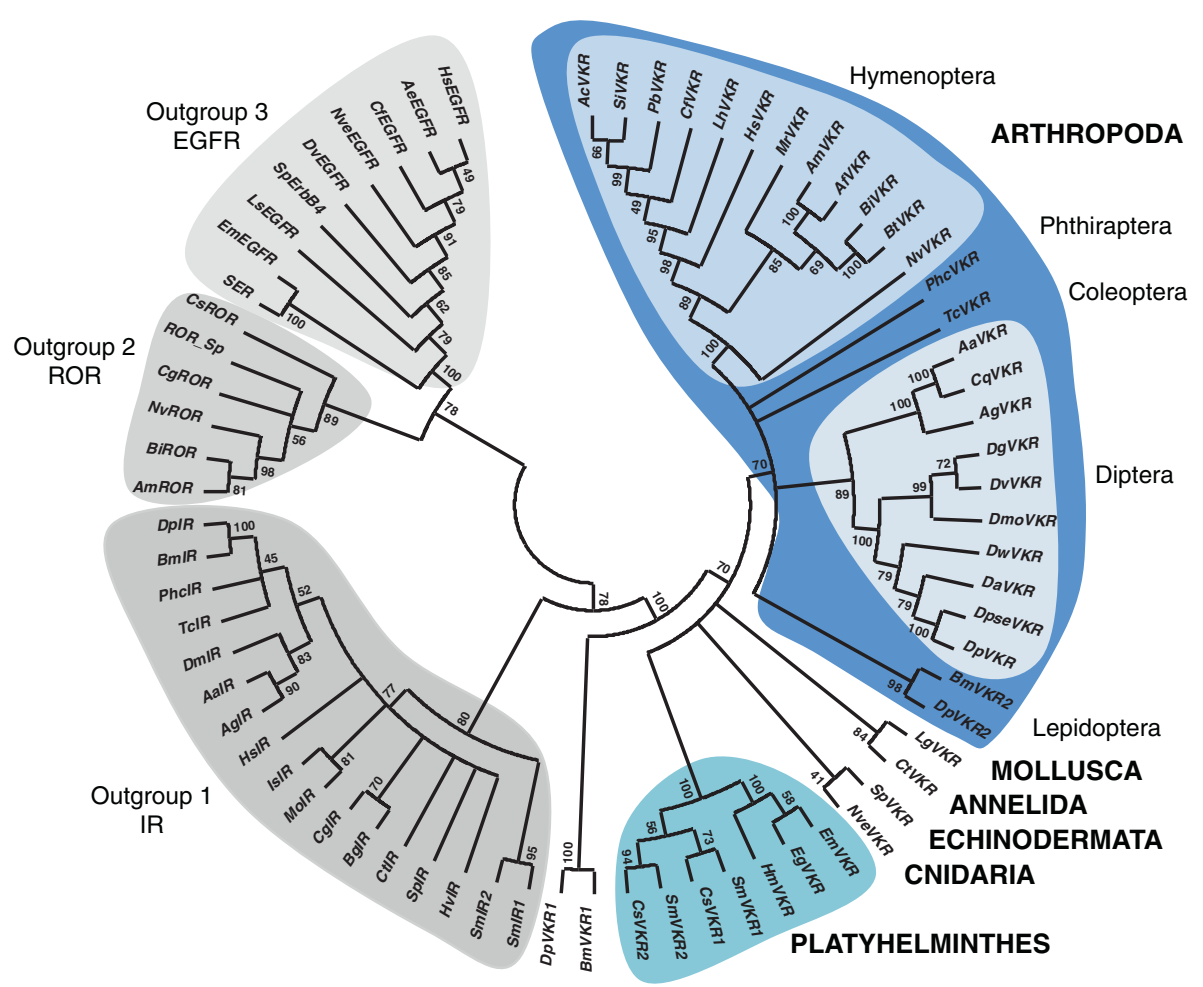

Figure 1 Phylogenetic construction of the VKR family. A maximum likelihood tree was generated from the 40 VKR sequences using MEGA5 under the $J T+G+I$ model with 100 bootstrap repetitions. Outgroups are formed by insulin receptors (IR, outgroup 1), RTK-like orphan receptors (ROR, outgroup 2) and/or EGF receptors (EGFR, outgroup 3) of the following species: A. aegypti (AalR, AAB17094.1), A. echiniator (AeEGFR, EGl67610.1), A. gambiae (AgIR, EAA00322.3), A. mellifera (AmROR, XP_397058.4), B. impatiens (BiROR, XP_003490221.1), Biomphalaria glabrata (BgIR, AAF31166.1), B. mori (BmIR, NP_001037011.1), C. floridanus (CfEGFR, EFN60989.1), Crassostrea gigas (CgIR, EKC21734.1; CgROR, EKC27495.1), C. sinensis (CsROR, GAA34401.2), C. teleta (CtIR, ELT96360.1), D. melanogaster (DmIR, AAC47458.1), D. plexippus (DplIR, EHJ65074.1), D. virilis (DvEgfr, ABD64816.1), E. multilocularis (EmEGFR, CAD56486.1), H. saltator (HsIR, EFN83767.1; HsEGFR, EFN75184.1) Hydra vulgaris (HvIR, Q25197.1), Ixodes scapularis (IIIR, XP_002416224.1), Lymnaea stagnalis (LsEGFR, ABQ10634.1), Metaseiulus occidentalis (MolR, XP_003739590.1), N. vitripennis (NvROR, XP_001601308.2; NvEGFR, XP_001602830.2), P. humanus corporis (PhcIR, XP_002430961.1), S. mansoni (SmIR1, GenBank: AAN39120.1; SmIR2, GenBank: AAV65745.2 and SER, GenBank: AAA29866.1), S. purpuratus (SpIR, XP_784376.3; SpErbB4, XP_791361.3 and ROR_Sp, XP_003729469.1) and T. castaneum (TCIR, EFA11583.1). For VKR abbreviations and accession numbers, see Table 1.

tree. Finally, the cnidarian (NveVKR), annelid (CtVKR), mollusc (LgVKR) and echinoderm (SpVKR) proteins were correctly bound to the VKR tree, but no conclusion can be drawn about their phylogenetic proximity since only one $v k r$ is available for each phylum. Thus, in these studies, we have confirmed the distribution of VKR in arthropods (in insects particularly), platyhelminths and echinoderms and found new VKR orthologs in three additional phyla, Annelida (Capitella teleta), Mollusca (Lottia gigantea) and Cnidaria (N. vectensis) (Figure 2). Additionally, putative VKR sequences were found in EST databases of different orders of Arthropoda (Cochliomyia hominivorax (gb| FG295125.1), Rhipicephalus microplus (gb|FG302900.1), Coptotermes formosanus (GI:345171826) and Crassostrea gigas (GI:313329111)), in the mollusc Biomphalaria glabrata (Contig1132.1, Biomphalaria glabrata Genome Initiative, biology.unm.edu/biomphalaria-genome/index.html), in the annelid Helobdella robusta (gb|EY370614.1) and in the echinoderm Paracentrotus lividus ( emb|AM524433.1).
The identification of a putative VKR in this second echinoderm indicates that $v k r$ genes could be present in multiple deuterostomes, and excludes a recent horizontal gene transfer or a genomic material contamination. The presence of VKR in Cnidaria, an animal lineage early diverging from Bilateria, suggests that the VKR family emerged prior to the expansion of Bilateria, the clade that comprises almost all extant animals. However, though we identified NveVKR in the anthozoan Nematostella, we could not detect any VKR sequence in the genome of the hydrozoan Hydra magnipapillata, a result in agreement with the recent genome-wide RTK screening performed for this species [15]. Such a difference could be related to those already described for gene diversity and content between anthozoan and hydrozoan genomes [16]. At this time, cnidarians are the first branch of Metazoa in which $v k r$ genes have been found and the question of their presence or not throughout all phyla of Bilateria is still open. Extensive research in vertebrate and roundworm genomes allowed us 


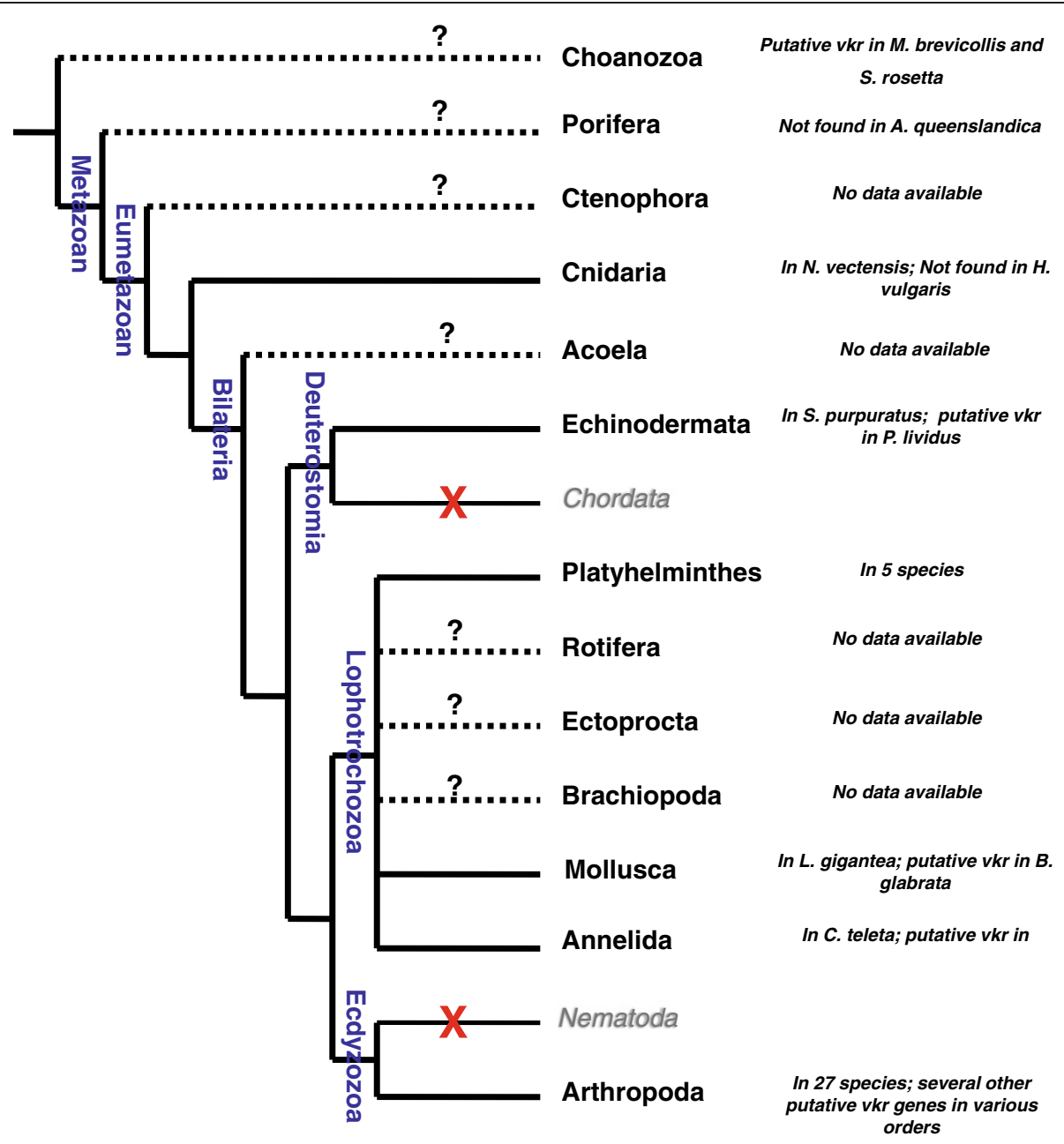

Figure 2 Distribution of vkr genes in metazoan phyla. Vkr genes were definitely identified in Arthropoda (27 insect species), Platyhelminthes (trematode and cestodes parasites), and in Mollusca, Annelida and Echinodermata (at least in one species for each phylum). Additionally, putative VKR sequences have been detected in the genomes of many other arthropods, in the mollusc Biomphalaria glabrata, in the annelid Helobdella robusta and in the echinoderm Paracentrotus lividus. The presence of VKR has been confirmed in the cnidarian N. vectensis but not found in Hydra (H. magnipapillata and H. vulgaris) genomes. The "unclassified" TK (UTK12) [5] and the RTKS kinase [17] found respectively in the choanoflagellates Monosiga brevicollis and Salpingoeca rosetta possess an architecture similar to VKR proteins. However, no vkr gene was found in the poriferan Amphimedon queenslandica. Concerning Ctenophora, Acoela, Rotifera, Ectoprocta, Brachiopoda phyla, genomic data are currently not sufficient to assess the presence or not of $v k r$ genes in these organisms. No $v k r$ genes were found in Chordata (vertebrates) nor in Nematoda (worms).

to conclude that VKR is absent from Chordata and Nematoda but the lack of genomic data for species of the Acoela, Rotifera, Ectoprocta and Brachiopoda phyla still stands in the way of a better understanding of the place of VKR throughout evolution (Figure 2). Moreover, the existence of $v k r$ in Premetazoa remains to be considered because we have recently noticed in choanoflagellates that the "unclassified" TK annotated as UTK12 of Monosiga brevicollis [5] as well as the RTKS kinase of Salpingoeca rosetta [17] have both a protein architecture similar to that of VKR proteins.

\section{Diversification of $v k r$ genes}

Previous studies have already demonstrated that $v k r$ genes were substantially heterogeneous in length as well as in intron-exon composition throughout species, while their organization within a given order was rather well conserved, like in Diptera [12]. In this work, we have made an extensive study of the exon/intron structure of all the discovered $v k r$ genes using the GenScan [18] and Augustus [19] gene prediction Web servers. Data in Table 2 illustrate the gene composition of $v k r$ genes from all the species grouped into families. They indicate for each $v k r$ gene the total number of exons and specify the exons that encode VFT, TM or TK domains. The results confirmed a wide heterogeneity for $v k r$ genes across the diverse phyla. In arthropods, $v k r$ genes have highly variable size (estimated from 4 to $65 \mathrm{~kb}$ ) and exon numbers (from 5 in Drosophilidae and Culicidae to 13 
Table 2 Characteristics and structural organization of $\mathbf{v k r}$ genes

\begin{tabular}{|c|c|c|c|c|c|c|}
\hline \multirow[t]{2}{*}{ Origin } & \multirow[t]{2}{*}{ Name } & \multirow[t]{2}{*}{ Size } & \multirow{2}{*}{$\begin{array}{l}\text { Exon } \\
\text { number }\end{array}$} & \multicolumn{3}{|c|}{ Coding exons for: } \\
\hline & & & & $\overline{\text { VFT }}$ & TM & TK \\
\hline
\end{tabular}

Cnidaria

Anthozoa

Edwardsiidae

$\begin{array}{llllll}\text { Nvevkr } & 13,5 \mathrm{~kb} & 15 & \text { E2-E7 } & \text { E12 } & \text { E12-E15 }\end{array}$

Arthropoda

Diptera

Drosophilidae

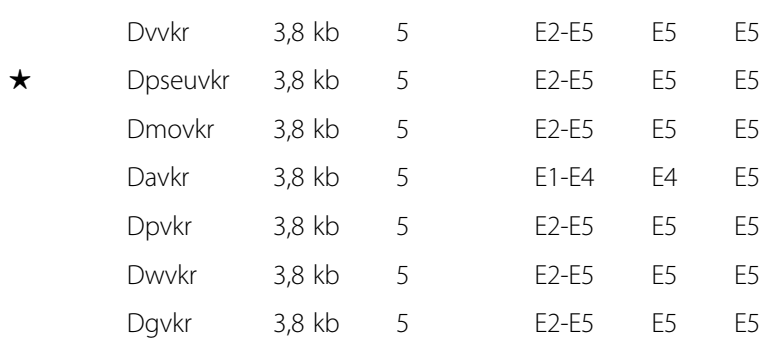

Culicidae

$\begin{array}{llllll}\text { Cqvkr } & 18 \mathrm{~kb} & 5 & \text { E2-E5 } & \text { E5 } & \text { E5 } \\ \text { Aavkr } & 48 \mathrm{~kb} & 5 & \text { E2-E5 } & \text { E5 } & \text { E5 }\end{array}$

Anophilinae

$\begin{array}{llllll}\begin{array}{lllll}\text { A Agvkr } \\ \text { Hymenoptera }\end{array} & 65 \mathrm{~kb} & 9 & \text { E6-E9 } & \text { E9 } & \text { E9 } \\ \text { Formicidae } & & & & & \\ \text { Pbvkr } & 6,5 \mathrm{~kb} & 11 & \text { E4-E8 } & \text { E8 } & \text { E9-E11 } \\ \text { Sivkr } & 5,5 \mathrm{~kb} & 9 & \text { E4-E6 } & \text { E8 } & \text { E8-E9 } \\ \text { Hsvkr } & 7 \mathrm{~kb} & 11 & \text { E4-E7 } & \text { E7 } & \text { E8-E11 } \\ \text { Acvkr } & 7 \mathrm{~kb} & 11 & \text { E4-E6 } & \text { E6 } & \text { E7-E11 } \\ \text { Lhvkr } & 6,5 \mathrm{~kb} & 11 & \text { E3-E5 } & \text { E5 } & \text { E6-E7 } \\ \text { Cfvkr } & 8 \mathrm{~kb} & 11 & \text { E4-E8 } & \text { E8 } & \text { E8-E9 } \\ \text { Aevkr } & 15,5 \mathrm{~kb} & 13 & \text { E3-E6 } & \text { E6 } & \text { E7-E10 }\end{array}$

Apidae

$\begin{array}{llll} & \text { Afvkr } & 8,5 \mathrm{~kb} & 12 \\ & \text { Amvkr } & 8 \mathrm{~kb} & 11 \\ \text { Bivkr } & 37 \mathrm{~kb} & 12 \\ \text { Btvkr } & 37 \mathrm{~kb} & 12\end{array}$

Megachilidae

\begin{tabular}{|c|c|c|c|c|}
\hline Mrvkr & $5 \mathrm{~kb}$ & 8 & E2-E5 & E5 \\
\hline Pteromalidae & & & & \\
\hline Nvvkr & $27 \mathrm{~kb}$ & 12 & E6-E11 & E12 \\
\hline
\end{tabular}

Lepidoptera

Bombycidae

$\begin{array}{llllll}\text { Bmvkr1 } & 24 k b & 5 & \text { E5 } & \text { E5 } & \text { E5 } \\ \text { Bmvkr2 } & 7 \mathrm{~kb} & 5 & \text { E2-E4 } & \text { E4 } & \text { E4 }\end{array}$

Table 2 Characteristics and structural organization of $\mathbf{v k r}$ genes (Continued)

Nymphalidae

$\begin{array}{llllll}\text { Dplvkr1 } & 7 \mathrm{~kb} & 6 & \text { E4-E5 } & \text { E5 } & \text { E5-E6 } \\ \text { Dplvkr2 } & 21,5 \mathrm{~kb} & 8 & \text { E3-E7 } & \text { E7 } & \text { E7-E8 }\end{array}$

Coleoptera

Tenebrionidae

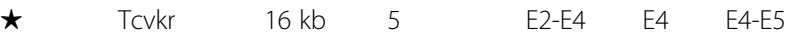

Phthiraptera

Pediculidae

$\begin{array}{lllll}\text { Phcvkr } & 9,5 \mathrm{~kb} & 10 & \text { E4-E8 } & \text { E8 }\end{array}$

Annelida

Polychaeta

Capitellidae

$\begin{array}{llllll}\text { Ctvkr } & 8,5 \mathrm{~kb} & 18 & \mathrm{E} 4-\mathrm{E} 11 & \mathrm{E} 12 & \mathrm{E} 12\end{array}$

Mollusca

Gasteropoda

Lottiidae

$\begin{array}{llllll}\text { Lgvkr } & 9,5 \mathrm{~kb} & 17 & \mathrm{E} 4-\mathrm{E} 10 & \mathrm{E} 11 & \mathrm{E} 11-\mathrm{E} 15\end{array}$

Platyhelminths

Trematoda

Schistosomatidae

$\begin{array}{lllllll}\star & \text { Smvkr1 } & 30 \mathrm{~kb} & 16 & \text { E7-E10 } & \text { E10 } & \text { E11-E14 }\end{array}$

* Smvkr2 $30 \mathrm{~kb} \quad 18 \quad$ E7-E10 E10 E11-E14

Opistorchiidae

$\begin{array}{llllll}\text { Csvkr1 } & 48 \mathrm{~kb} & 15 & \text { E5-E9 } & \text { E9 } & \text { E10 } \\ \text { Csvkr2 } & 34 \mathrm{~kb} & 15 & \text { E4-E8 } & \text { E8 } & \text { E8-E11 }\end{array}$

Cestoda

Taeniidae

$\begin{array}{llllll}\text { Egvkr } & 23,5 \mathrm{~kb} & 15 & \text { E3-E7 } & \text { E9 } & \text { E10-E12 } \\ \text { Emvkr } & 19 \mathrm{~kb} & 16 & \text { E4-E8 } & \text { E10 } & \text { E11-E13 }\end{array}$

Hymenolepididae

Hmvkr $19 \mathrm{~kb} \quad 11 \quad$ E1-E6 E7 E8-E10

Echinodermata

Echinoida

Strongylocentrotidae

* Spvkr $60 \mathrm{~kb} \quad 21 \quad$ E9-E14 E16 E17-E21

Data illustrate the composition of $v k r$ genes from all the animal species (see Table 1) which have been classified into their respective phylum, order and family. They indicate for each $v k r$ gene the number of exons and specify those that encode VFT, TM or TK domains. Stars indicate genes for which the complete CDNA sequence was obtained.

in the ant Acromyrmex echiniator). As it was already observed in the case of dipterans [12], sizes and/or exon-intron structures were homogeneous inside of the hymenopteran order, particularly for Formicidae and Apidae. The low number of exons found in $v k r$ genes of Drosophilidae and Culicidae could be due to the high 
degree of intron loss that has occurred during the evolution of the protostome lineage leading to flies and mosquitoes [20].

$V k r$ genes found in lophotrochozoan organisms (annelids, molluscs, platyhelminths), are overall more complex (15 to 18 exons) than the insect ones, except in the cestode $H$. microstoma (11 exons). Lophotrochozoan $v k r$ genes are according to this more similar to that one detected in the phylogenetically basal animal $N$. vectensis. Indeed, Nvevkr is also intron-rich (15 exons), respecting therefore the known high complexity of the genes present in early animal genomes [20]. Spvkr found in the echinoderm $S$. purpuratus remains the most complex $v k r$ gene found with a size of $60 \mathrm{~kb}$ and a total of 21 exons. In trematodes, the organisation of $S m v k r 1$ and Smvkr2 genes was shown to be quite identical (see Table 2, and [14]), arguing for a duplication event in S. mansoni.

As a preliminary approach to understand $v k r$ gene evolution, we have analysed the conservation of intron positions throughout the diverse animal groups (Figure 3). First results overall indicated that $v k r$ genes were all sharing at least one conserved intron position, arguing that they might belong to the same family.

We also noticed that 12 out of 14 intron positions of Nvevkr were found in at least one phylum and that most of these conserved exon-intron boundaries were located in regions that encode VFT and TK domains. Most of these positions were shown to be conserved in the annelid (Ctvkr), mollusc (Lgvkr) and echinoderm (Spvkr) genes. Inversely, only a very limited number of intron positions seem to be conserved in arthropods as well as in platyhelminthes, indicating a profound reorganization of $v k r$ genes along evolution. Finally, specific or nonconserved intron positions were also found in various $v k r$ genes (Figure 3). Taken together, these studies demonstrate that $v k r$ genes are highly variable in size and in complexity but that, in spite of their heterogeneity, all of them possess common features, which are conserved from Cnidaria to the other phyla.

\section{Conservation of VKR tyrosine kinase domains}

Using the multiple alignment ClustalW algorithm, we have compared the TK domain sequences of the 40 VKRs and generated an identity matrix. As it could be expected for catalytic structures, the sequences are relatively well conserved across all species, with the best scores of identity observed between the species belonging to a same order. For example, in Hymenoptera the TK domains of Bombus and Apis species are more than 92\% identical, in Diptera, those of Drosophila species are more than $75 \%$ identical and those of the mosquitoes A. gambiae and A. aegypti are $93 \%$. For platyhelminth VKRs, identities between TK domains scored between
57 to $96 \%$, with the best score registered between the two cestode parasites E. multilocularis and E. granulosus. In Lepidoptera, the TK domains of Bombyx and Danaus VKRs were less conserved, except for BmVKR2 and DplVKR2 that share $81 \%$ of identity.

In the aligned sequences, we could identify most of the residues shown to be essential for TK activity [21]. As indicated in Figure 4, the glycine-rich motif

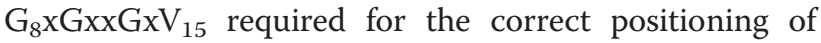
ATP is found in all VKRs except in that of D. ananassae, which lacks the first $G$ residue. The $V_{32} A x K(16 x) E_{52}$ motif essential for ATP stabilization is also tightly conserved, except for the hymenopteran $N$. vitripennis VKR. The phosphotransfer site $\mathrm{H}_{147} \mathrm{RD}(\mathrm{L} / \mathrm{V} / \mathrm{I}) \mathrm{xxRNxL}_{156}$ is also present in the catalytic loop of all VKRs but in the insect VKRs of P. humanus corporis and $H$. saltator, this motif is interrupted by an insertion. Without exception, all VKRs possess the $\mathrm{D}_{194} \mathrm{FG}_{196}$ site essential for the binding to the catalytic magnesium ion. In the activation loop, the two juxtaposed $\mathrm{Y}_{211} \mathrm{Y}_{212}$ autophosphorylation site that allows an open access to ATP and substrates in many activated RTKs (like insulin receptors) [22], is found in all VKRs, except in VKR1 isoforms of B. mori and $D$. plexippus in which a single $\mathrm{Y}$ is found at this position. The $\mathrm{M}_{262}(\mathrm{~A} / \mathrm{S}) \mathrm{PE}_{265}$ motif implicated in the stabilization of the active kinase core is present in most VKRs, but the A/S residue is replaced by a $\mathrm{P}$ residue in all the Drosophila species as well as in cestodes. We can note also that this motif is totally absent from the VKR of the ant L. humile. Other hydrophobic residues $M_{56}$, $\mathrm{L}_{67}, \mathrm{~L}_{91},(\mathrm{~L} / \mathrm{V} / \mathrm{I})_{284},(\mathrm{~L} / \mathrm{V} / \mathrm{I})_{288}$, that compose in every active kinase spatially conserved motifs, termed spines, and that play a major role in kinase dynamic assembly and activity [23], are present in all VKRs. Overall, these data showed that a large part of VKR sequences contained all the motifs essential for TK activity. Furthermore, our previous demonstrations $[12,14]$ that recombinant receptors from $A$. mellifera and $S$. mansoni were catalytically active and able to autophosphorylate, strongly suggest that most of the VKRs identified in this work might exhibit similarly kinase activity. Concerning the ones (DaVKR, NvVKR, BmVKR2, DplVKR2, PhcVKR, HsVKR and LhVKR), that lack one or several motifs essential for catalytic activity, we could suggest that they constitute dead or pseudokinases, but this conclusion should be taken with much caution because of possible artefacts in gene prediction or sequences. Further analyses of their kinase potential would be needed to conclude.

\section{Conservation and divergence of VKR ligand-binding domains}

Multiple alignment of the VFT module sequences from the 40 VKRs shows that protein sequences are relatively 


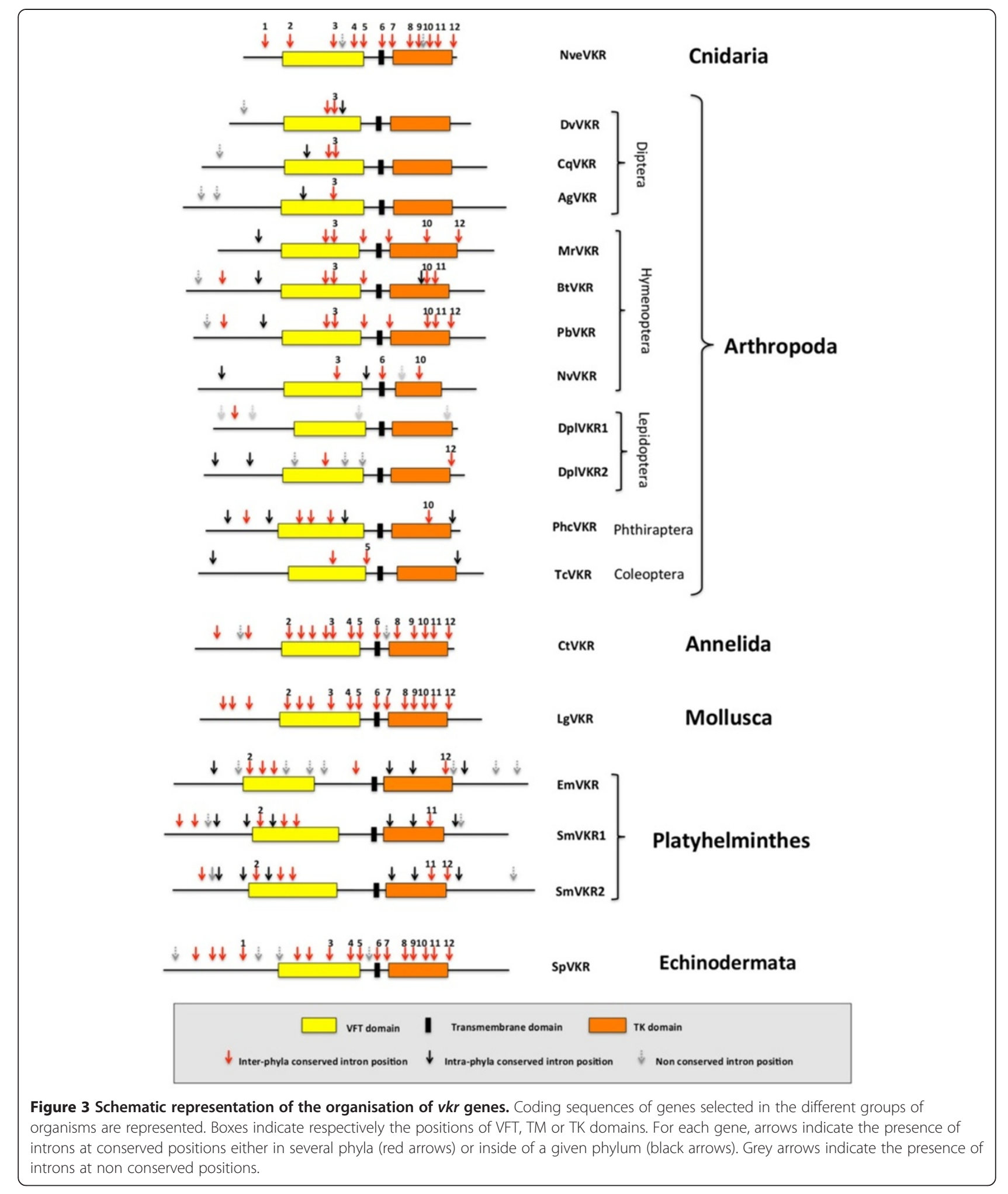

well conserved, particularly in insect species (Figure 5). Excluding the two lepidopteran VKRs, all insect VFT sequences share from 29\% (between PhcVKR and DpseVKR) to $98 \%$ (between the two Bombus species) of identity. Interestingly, VFT sequences are highly conserved within a given order. As an example, VKRs from the 13 hymenopteran species all share at least 70\% identity. Likewise, there is at least $50 \%$ identity between the 


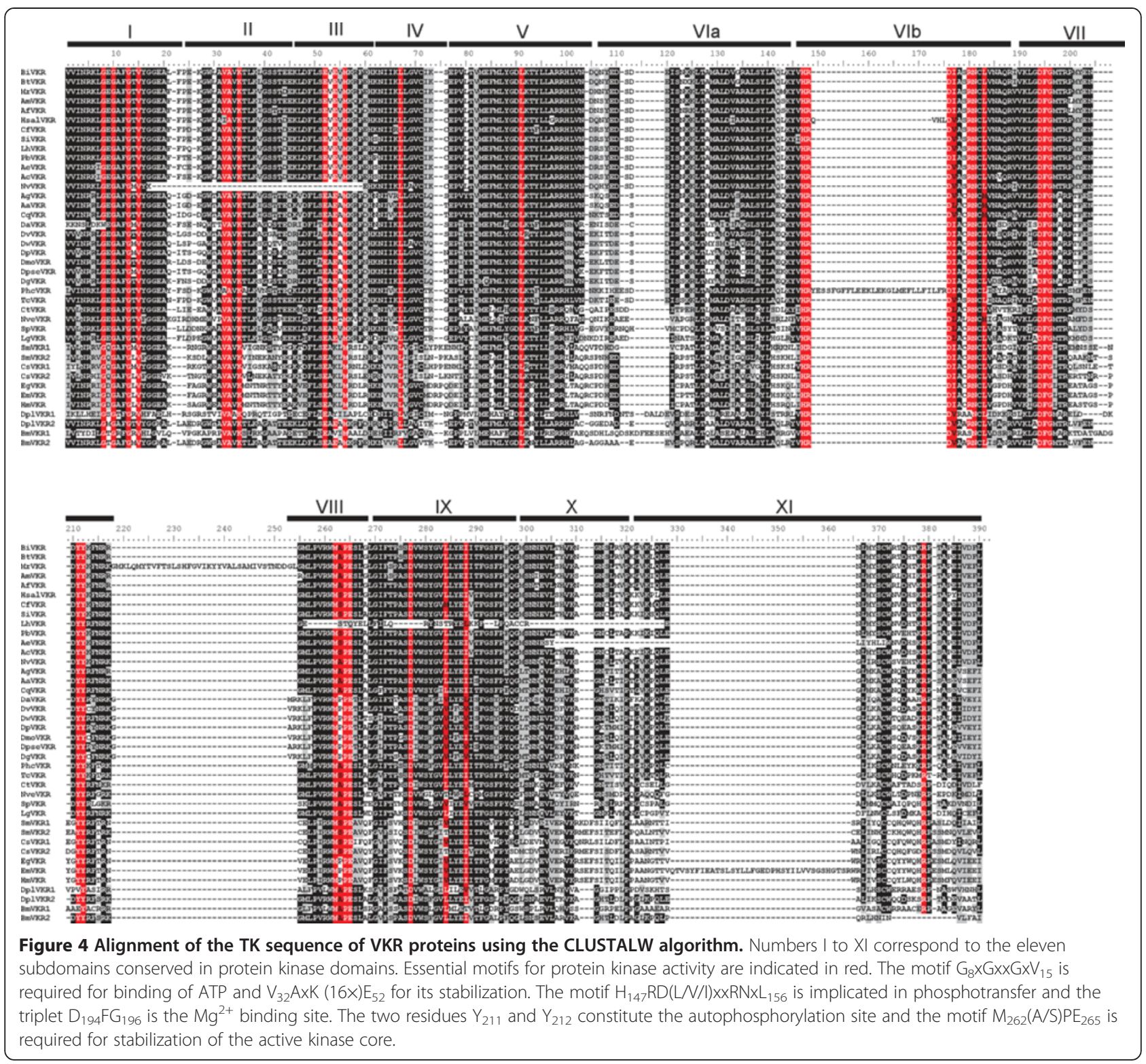

VKRs identified in all dipteran species. Taken together, these results agree with an evolutionary conservation of insect VFT sequences. However, an exception concerns the lepidopteran VKRs (BmVKR1/2 and DplVKR1/2) for which VFT module sequences are highly divergent from each other and from all insect VFT domains (less than $16 \%$ of identity). Still, the BmVKR2 VFT module seems closer to that of DplVKR2 ( $40 \%$ of identity). Concerning the VFT sequences of platyhelminths, intermediate levels of conservation were observed between trematode and cestode sequences (24\% to $44 \%$ identity) while we could note, as previously observed for the TK domain, a marked identity (93\%) within the genus Echinococcus between EgVKR and EmVKR VFT domains. Finally, other VKRs (from Annelida, Mollusca and Cnidaria species) were found to be highly divergent from platyhelminth and lepidopteran VKRs in their VFT domains, but as discussed above concerning their phylogeny (Figure 1), available sequences are not sufficient to state about receptor evolution in these phyla.

VFT modules constitute the binding pocket of various receptors activated by small molecules [13]. They are composed of two lobes connected via flexible tethers that close around the bound ligand. In most class $C$ GPCRs, these modules contain the binding site for natural amino acids or derivatives and ligand recognition is dependent on a consensus motif of 8 residues that participate to the binding of the $\alpha$-amino acid functions (i.e. primary amine and carboxylic acid) [24]. Among these residues, the serine that binds the $\mathrm{COOH}$ group of 


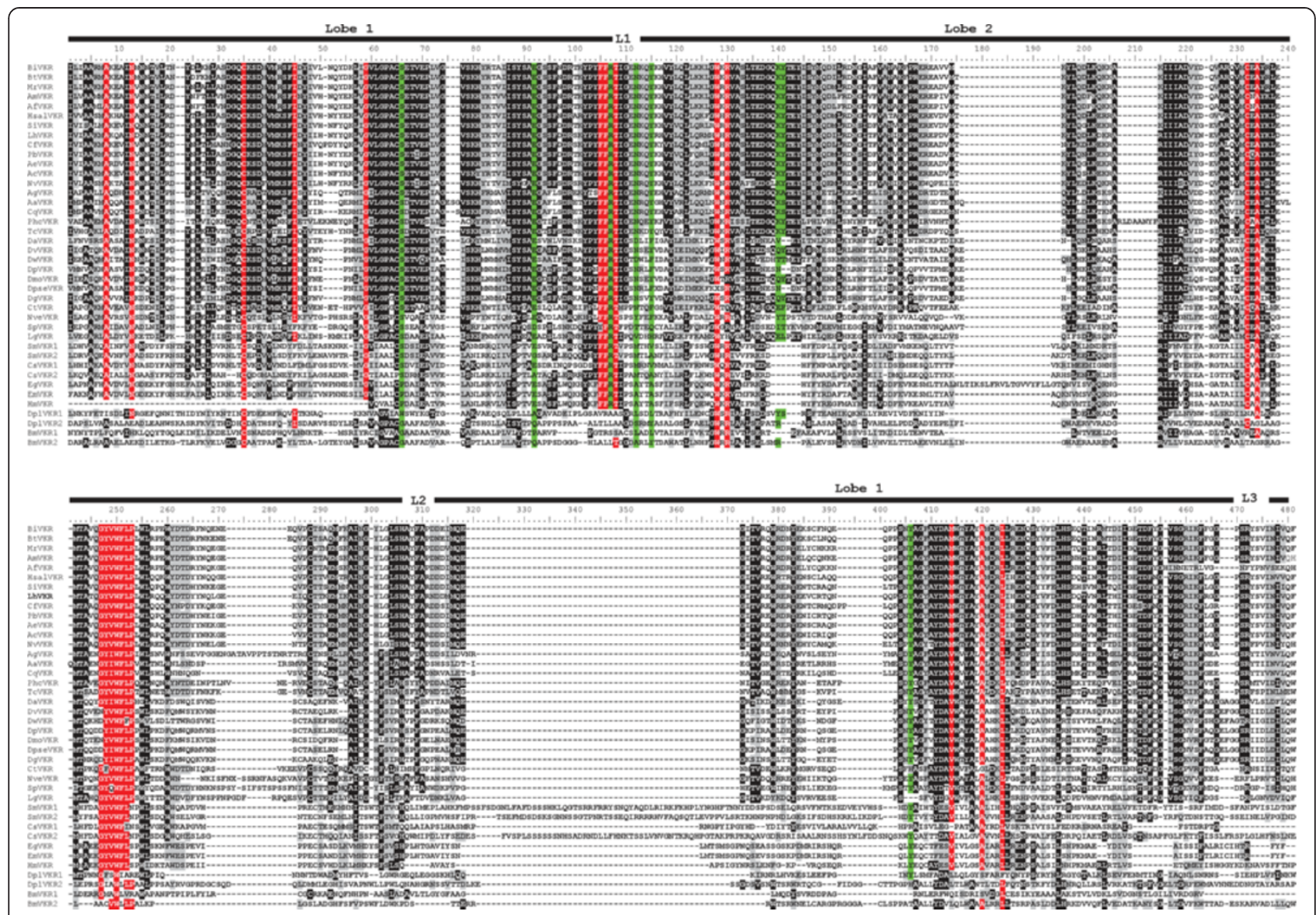

Figure 5 Alignment of the VFT sequence of VKR proteins using the CLUSTAL W algorithm. Lobe I and Lobe II (indicated by the upper black line) and the three linkers (L1, L2, L3) constitute the structure of VFT domains. Residues highlighted in red are highly conserved in all or most VKR sequences, and residues highlighted in green correspond to the consensus amino-acid binding motif of VFT domains [24].

glutamate in mGluR1 ( $\left.\operatorname{Ser}_{165}\right)$, is the most conserved residue in class C GPCRs. In most VKRs (except in cestode and lepidopteran receptors), this residue is strictly conserved (at position 66 in the VFT sequence, Figure 5). The $\mathrm{R}_{107}$, another important residue composing the consensus site for AA binding in VFT modules [24] is also present in all VKRs (except in Lepidoptera). These observations are totally in agreement with our demonstration that amino-acids, and particularly L-arginine, are able to bind and activate both honey bee and schistosome VKRs $[12,14]$ and we have recently confirmed the requirement of $S_{66}$ and $R_{107}$ for the amino-acid recognition by VKR receptors (unpublished). Interestingly, if the six other elements composing the consensus motif in VFT modules for amino acid binding [24] are different in the ligand-binding domains of VKRs, we could note that at their exact positions, many VFT domains present identical residues (highlighted in green in Figure 5), and this is particularly obvious inside of the Hymenoptera order. Among these residues, $\mathrm{Y}_{406}$ was found to be present in all VKRs (except in lepidopteran sequences). $\mathrm{Y}_{406}$ is exactly at the position $\mathrm{K}_{509}$ in mGluR1, the residue involved in the binding of the terminal carboxylic group of glutamate [12], and this strengthens our observation that glutamate is not a ligand for VKR. Additionally, we can see in the VFT alignment several conserved residues and motifs (highlighted in red, Figure 5), such as $\mathrm{C}_{35}$ and $\mathrm{W}_{128}$ which are present in all domains and the motif $\left(\mathrm{G}_{247} \mathrm{Y}(\mathrm{V} / \mathrm{I}) \mathrm{WFLPxWL} \mathrm{L}_{256}\right)$ which is present in most VKRs. Finally to investigate the potential importance of these conserved residue positions for the VFT properties, we performed a comparative modeling of the VFT domain of VKR using the ModWeb server of the ModBase databases. A VFT model of AmVKR was generated with the human Glutamate Receptor 5 (mGluR5) as homologous template (PDB: 3lmkA) (Figure 6). Results confirmed the position inside of the ligand pocket formed between the two lobes of the VFT, of the residues potentially involved in amino-acid recognition. The model also indicates that many of the other conserved residues are constituents of alpha-helices or beta-sheets, and thus take part very likely in the tertiary structure of the domain. Interestingly, two residues $T_{108}$ and $\mathrm{I}_{109}$, highly conserved in most VKRs, are located in 


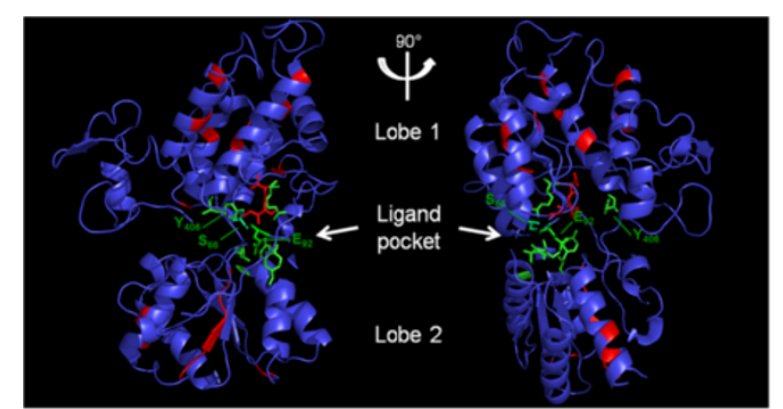

Figure 6 Evolutionary conservation of residues of the VFT domain visualized on the comparative modeling of AmVKR based on mGluR5 crystal (PBP: 3lmkA). Structurally important motifs are indicated in red and residues composing the consensus amino-acid binding motif of VFT domains [24] are in green sticks (for details see Figure 5). The highly conserved residues $S_{66}, E_{92}$ and $\mathrm{Y}_{406}$, found to be important for amino-acid binding, are localized within the binding pocket, together with two conserved structural residues $T_{108}$ and $\mathrm{I}_{109}$ shown in red sticks.

the putative ligand binding pocket, suggesting that they could contribute with the conserved residues of the amino-acid binding motif to ligand specificity and/or affinity. Taken together, these results let suppose that in spite of a partial conservation of primary structures, the VFT domains of many VKRs can bind a common ligand.

\section{Conclusions}

This survey based on the analysis of newly released genomic data has allowed us to show that $v k r$ genes actually represent a novel RTK family, widespread in the bilaterian branch of Eumetazoa. From this study we can extend the presence of VKR to a large variety of protostomes (Ecdysozoa and Lophotrochozoa). However, after the analysis of a large number of deuterostome genomes (Chordata and Hemichordata), it seems that deuterostomes would not contain $v k r$ genes, with the exception of echinoderms. In these studies, an important information concerns the detection of NveVKR in the basal metazoan $N$. vectensis. From the presence of a $v k r$ gene in Cnidaria, we can suggest that the origin of VKR would be anterior to the radiation of Bilateria, and possibly close in time to that of the setting-up of animal multicellularity. This would agree with the general acceptance that emergence of a series of cell surface receptors (including RTKs) necessary for cell adhesion, differentiation and cell-cell communications has driven evolution towards multicellularity [6]. In this context, we have recently found putative RTKs exhibiting an architecture close to that of VKR proteins in the genomes of the choanoflagellates $M$. brevicollis [5] and S. rosetta [17], which are free-living unicellular and colonial flagellates considered to be the closest living relatives of the animals. These findings (unpublished) encourage us to postulate that VKR could represent an ancient RTK present early in protists, that might have contributed to the establishment of multicellularity and to animal development.

Another important question concerns the distribution and stability of VKR throughout evolution. $V k r$ genes were found preferentially in the genomes of protostomes and particularly in insect genomes, but the large number of insect VKR sequences could likely result from the relative abundance of insect sequences in genomic databases. However, it was very interesting to observe that the finding of one $v k r$ in all species of a given genus is not a general rule. For example, in the Drosophila genus, D. melanogaster and some others exceptionally do not possess a $v k r$ gene [12]. Also, it is surprising that no $v k r$ exists in other ecdysozoa like nematodes, and in $C$. elegans particularly.

In this work, we have analysed the exon/intron structure of all $v k r$ genes and shown that their organization is widely heterogeneous across the different phyla. However, all $v k r$ genes share intron positions common to the ancestral gene $N v e v k r$, and this suggests that they might have been derived from this common ancestor, then subjected to more or less marked reorganization along evolution. Finally, the question of the existence of two $v k r$ genes in Trematoda and Lepidoptera is still open, together with the problem of the "keep or loss" of $v k r$ in some species. Further investigations about the functions of VKR in the biology and physiology of organisms should be required to answer these questions.

We have previously shown that $v k r$ genes were preferentially expressed in larval stages and in gonads of several organisms, including sea urchin, mosquito and the trematode $S$. mansoni, thus suggesting a role of the receptor in embryogenesis and gonad development $[11,12,14]$. About their functional activity, we have been able to demonstrate the tyrosine kinase activity of several VKRs $[12,14]$ and sequence information here obtained for the TK domains of all VKRs confirms that most VKRs should be active kinases as well. VKR receptor activation was shown to be dependent on the binding of L-amino-acids, and specifically of L-arginine, to the extracellular VFT domain, in which conserved residue positions (for example $\mathrm{S}_{66}$ and $R_{107}$ ) are essential for ligand-receptor interaction and probably involved in specificity and affinity of L-arginine. However, in some VKRs, these major conserved residues are not present, suggesting that they could bind other ligands and perhaps have different functional activities. We have shown that SmVKR1 and SmVKR2 of S. mansoni differ both in the primary sequence of their VFT domain and in their localization in the parasite. The observation that these receptors are activated respectively by L-arginine and Calcium [14] really illustrates this possible functional divergence between VKR. 
Currently, diverse strategies are developed to analyse the consequences of VKR knock-down in organisms. Preliminary results of VKR targeting by RNA interference in $S$. mansoni have confirmed the importance of SmVKR1 and SmVKR2 for growth and differentiation of reproductive organs and parasite fertility (to be published). The use of other organisms as candidate models for studying the function of VKR is under investigation.

In conclusion, the VKR family is a little-known RTK family that deserves to be further explored in order to determine more precisely its evolutionary origin, its possible importance for the emergence and specialization of Metazoa, and to understand how its maintenance or its loss in various phyla or species could be in relation with development and physiological activities (like reproduction).

\section{Methods}

\section{Genome database searches}

Putative $v k r$ sequences were searched using tBLASTn on genomic sequences available in the following databases: GenBank (http://www.ncbi.nlm.nih.gov/blast/Blast.cgi), FlyBase (http://flybase.org/), VectorBase (http://www. vectorbase.org), Wellcome Trust Sanger institute databases (http://www.sanger.ac.uk/resources/databases/) and the JGI genome portal (http://genome.jgi-psf.org/). Additionally, we also searched for $v k r$ sequences in Hydra magnipapillata (hydrazome), Schmidtea mediterranea (SmedDB) and Schistosoma japonicum(schistoDB) genome databases.

Selected genomic sequences were analysed by GenScan (http://genes.mit.edu/GENSCAN.html) and Augustus (http://bioinf.uni-greifswald.de/augustus/) gene prediction servers, and putative VKR proteins were then determined. The presence of VFT, TM and TK domains was verified, and their delimitation defined using the SMART software (http://smart.embl-heidelberg.de/). Finally, TM regions were confirmed with the TMHMM server (http://www. cbs.dtu.dk/services/TMHMM).

\section{Phylogenetic analyses}

Protein sequences (listed in Additional file 1) were aligned using ClustalW algorithm in the BioEdit v7.1 software, and manually corrected. Maximum likelihood trees were built using MEGA5 [25] under the JTT+I+G model, with 100 bootstrap repetitions.

\section{Comparative modeling}

Comparative modeling was performed on the VFT sequence domain of VKRs using the comparative modeling web-server Modweb (https://modbase.compbio.ucsf.edu/ scgi/modweb.cgi) of the ModBase databases. Calculation and evaluation of models were performed with ModPipe software using sequence-sequence, sequence-profile and profile-sequence methods for fold assignment and targettemplate alignment.

\section{Additional file}

Additional file 1: FastA text file containing the full length sequences of the 40 VKR proteins aligned using the Clustal W algorithm. For VKR abbreviations, see Table 1.

\section{Competing interests}

The authors declare that they have no competing interests.

\section{Authors' contributions}

MV, NG and AA carried out research of genomic sequences in databases and sequence analysis using gene prediction servers. MV and NG performed functional annotation based on sequence similarity using SMART and sequence alignments of protein sequences. MV and MM performed phylogenetic studies. NG made comparative modeling experiments. JV and CD participated in the design of the experiments. CD coordinated the study. MV and CD wrote the manuscript. All the authors read and approved the final manuscript.

\section{Acknowledgements}

This research was supported by the Institut de la Sante et de la Recherche Medicale and Université Lille Nord de France. MV, NG and MM fellowships were from the Ministere de l'Education Nationale et de la Recherche, France. The authors wish to thank Dr R.J. Pierce for his helpful advices.

\section{Author details}

${ }^{1}$ Center for Infection and Immunity of Lille (CIIL), Inserm U1019, CNRS-UMR 8204, Institut Pasteur de Lille, 59019, Lille, France. ${ }^{2}$ Institut de Génétique et de Biologie Moléculaire et Cellulaire (IGBMC), Inserm U964, CNRS-UMR 7104 Université de Strasbourg, 67404, IIIkirch, CU Strasbourg, France. ${ }^{3}$ UMR8161 CNRS, Institut de Biologie de Lille, 1, rue du Pr. Calmette BP 467, 59021, Lille Cedex, France.

Received: 23 November 2012 Accepted: 24 May 2013 Published: 30 May 2013

\section{References}

1. Lemmon MA, Schlessinger J: Cell signaling by receptor-tyrosine kinases. Cell 2010, 141:1117-1134.

2. Schäcke H, Schröder HC, Gamulin V, Rinkevich B, Müller IM, Müller WEG: Molecular cloning of a tyrosine kinase gene from the marine sponge Geodia cydonium: a new member belonging to the receptor tyrosine kinase class II family. Mol Memb Biol 1994, 11:101-107.

3. Manning G, Whyte DB, Martinez R, Hunter T, Sudarsanam S: The protein kinase complement of the human genome. Science 2002, 298:1912-1934

4. King N, Carroll SB: A receptor tyrosine kinase from choanoflagellates: molecular insights into early animal evolution. Proc Natl Acad Sci USA 2001, 98:15032-15037.

5. Manning G, Young SL, Miller WT, Zhai Y: The protist, Monosiga brevicollis, has a tyrosine kinase signaling network more elaborate and diverse than found in any known metazoan. Proc Natl Acad Sci USA 2008, 105:9674-9679.

6. Suga H, Dacre M, De Mendoza A, Shalchian-Tabrizi K, Manning G, Ruiz-Trillo I: Genomic survey of premetazoans shows deep conservation of cytoplasmic tyrosine kinases and multiple radiations of receptor tyrosine kinases. Sci Signal 2012, 5(222):ra35.

7. Reidling JC, Miller MA, Steele RE: Sweet Tooth, a novel receptor proteintyrosine kinase with C-type lectin-like extracellular domains. J Biol Chem 2000, 275:10323-10330.

8. Morgan WR, Greenwald I: Two novel transmembrane protein tyrosine kinases expressed during Caenorhabditis elegans hypodermal development. Mol Cell Biol 1993, 13:7133-7143.

9. Manning G: Genomic overview of protein kinases. WormBook 2005, 13:1-9.

10. Robinson DR, Wu YM, Lin SF: The protein tyrosine kinase family of the human genome. Oncogene 2000, 19:5548-5557. 
11. Vicogne J, Pin JP, Lardans V, Capron M, Noël C, Dissous C: An unusual receptor tyrosine kinase of Schistosoma mansoni contains a Venus Flytrap module. Mol Biochem Parasitol 2003, 126:51-62.

12. Ahier A, Rondard P, Gouignard N, Khayath N, Huang S, Trolet J, Donoghue DJ, Gauthier M, Pin JP, Dissous C: A new family of receptor tyrosine kinases with a Venus Flytrap binding domain in insects and other invertebrates activated by aminoacids. PLoS One 2009, 4:e5651.

13. Pin JP, Galvez T, Prezeau L: Evolution, structure, and activation mechanism of family $3 / \mathrm{C}$ G-protein-coupled receptors. Pharmacol Ther 2003, 98:325-354.

14. Gouignard N, Vanderstraete M, Cailliau K, Lescuyer A, Browaeys E, Dissous C: Schistosoma mansoni: structural and biochemical characterization of two distinct Venus Kinase Receptors. Exp Parasitol 2012, 132:32-39.

15. Reddy PC, Bidayze SS, Ghaskadbi S: Genome-wide screening reveals the emergence and divergence of RTK homologues in basal Metazoan Hydra magnipapillata. J Biosci 2011, 36:289-296.

16. Putnam NH, Srivastava M, Hellsten U, Dirks B, Chapman J, Salamov A, Terry $A$, et al: Sea anemone genome reveals ancestral eumetazoan gene repertoire and genomic organization. Science 2007, 317:86-94.

17. Fairclough SR, Chen Z, Kramer E, Zeng Q, Young S, Robertson HM, Begovic $E$, et al: Premetazoan genome evolution and the regulation of cell differentiation in the choanoflagellate Salpingoeca rosetta. Genome Biol 2013, 14:R15.

18. Burge CB, Karlin S: Finding the genes in genomic DNA. Curr Opin Struct Biol 1998, 8:346-354.

19. Stanke M, Schöffmann O, Morgenstern B, Waack S: Gene prediction in eukaryotes with a generalized hidden Markov model that uses hints from external sources. BMC Bioinforma 2006, 7:62

20. Sullivan JC, Reitzel AM, Finnerty JR: A high percentage of introns in human genes were present early in animal evolution: evidence from the basal metazoan Nematostella vectensis. Genome Inform 2006, 17:219-229.

21. Hanks SK, Quinn AM, Hunter T: The protein kinase family: conserved features and deduced phylogeny of the catalytic domains. Science 1988, 241:42-52.

22. Zhang B, Tavare JM, Ellis L, Roth RA: The regulatory role of known tyrosine autophosphorylation sites of the insulin receptor kinase domain. An assessment by replacement with neutral and negatively charged amino acids. J Biol Chem 1991, 266:990-996.

23. Taylor S, Kornev AP: Protein kinases: Evolution of dynamic regulatory proteins. Trends Biochem Sci 2011, 36:65-77.

24. Acher FC, Bertrand HO: Amino acid recognition by Venus flytrap domains is encoded in an 8-residue motif. Biopolymers 2005, 80:357-366.

25. Tamura K, Peterson D, Peterson N, Stecher G, Nei M, Kumar S: MEGA5: molecular evolutionary genetics analysis using maximum likelihood, evolutionary distance, and maximum parsimony methods. Mol Biol Evol 2011, 28:2731-2739.

doi:10.1186/1471-2164-14-361

Cite this article as: Vanderstraete et al:: The venus kinase receptor (VKR) family: structure and evolution. BMC Genomics 2013 14:361.

\section{Submit your next manuscript to BioMed Central and take full advantage of:}

- Convenient online submission

- Thorough peer review

- No space constraints or color figure charges

- Immediate publication on acceptance

- Inclusion in PubMed, CAS, Scopus and Google Scholar

- Research which is freely available for redistribution

Submit your manuscript at www.biomedcentral.com/submit
Ciomed Central 